\title{
Growth conditions in infinitely generated groups
}

\author{
Avinoam Mann*
}

\begin{abstract}
We characterize groups in which each finitely generated subgroup has polynomial growth, under some uniformity conditions.
\end{abstract}

Mathematics Subject Classification (2000). 20F69.

Keywords. Polynomial growth.

We recall a few basic facts. Let $G$ be a finitely generated group, generated by a finite set $S$. Then each element $x \in G$ can be written as a product of elements from $S$ and their inverses. The least length of such an expression for $x$ is the length $l(x)$ of $x$. We write $s_{G}(n)=|\{x \in G \mid l(x) \leq n\}|$. This is the growth function of $G$, and $G$ has polynomial growth if there exist numbers $C$ and $d$ such that $s_{G}(n) \leq C n^{d}$. Strictly speaking, the growth function depends also on the set $S$. However, if the growth is polynomial with respect to one set of generators, it is polynomial with respect to any (finite) set of generators. In the sequel, whenever we say that a group $G$ has growth function $s_{G}(n)$, we mean that there exists some set of generators with respect to which this is the growth function. J. Wolf proved that virtually nilpotent groups have polynomial growth [Wo 68], and in a seminal paper M. Gromov proved the converse: all groups of polynomial growth are virtually nilpotent [Gr 81]. An alternative proof was given by v. d. Dries and Wilkie [VdDW 84a]. In the special case of linear growth, Justin [J 71] gave earlier an elementary proof that $G$ is virtually cyclic (see also [VdDW 84b]).

At the end of his paper, Gromov indicated a proof that a torsion-free group, all of whose finitely generated subgroups have polynomial growth, with a uniform bound on the degree, is also virtually nilpotent. In this paper we elaborate on that result, proving the following theorems.

\footnotetext{
* The author thanks the contributors to this issue, as well as the participants of the Asymptotic Group Theory meeting in Jerusalem in May 2006, for their many good wishes on the occasion of his retirement. $\mathrm{He}$ is also grateful to the referee for suggestions which improved the presentation of this note.
} 
Theorem 1. For a group $G$ the following two conditions are equivalent:

(i) There exist numbers $C$ and $d$, such that each finitely generated subgroup $H$ of $G$ satisfies $s_{H}(n) \leq C n^{d}$.

(ii) $G$ is virtually nilpotent and of finite rank.

Moreover, if (i) is satisfied, then $G$ is virtually nilpotent of class smaller than $\sqrt{2 d}$ and of rank at most $C$.

If we bound only the growth degrees, we obtain the result below. In its statement $h(d)$ denotes the maximal order of a finite subgroup of $\mathrm{GL}(d, \mathbb{Z})$.

Theorem 2. The following conditions on a group $G$ are equivalent:

(i) Each finitely generated subgroup has polynomial growth, and the growth degrees are bounded.

(ii) $G$ is locally Noetherian, and contains normal subgroups $N$ and $L$ such that $L \leq N, L$ is locally finite, $N / L$ is torsion free nilpotent of finite rank, and $|G: N|$ is finite.

Moreover, if (i) holds, with d being the bound on the growth degrees, then $N$ and $L$ can be chosen so that $N / L$ has rank at most $d$ and class less than $\sqrt{2 d}$, and $|G: N| \leq h(d)$.

Here we recall that $G$ has finite rank if there exists a number $r$ such that each finitely generated subgroup of $G$ can be generated by $r$ elements. The least such $r$ is termed the rank of $G$.

A simple example of a group of finite rank is the additive group of rational numbers. There each finitely generated subgroup is cyclic. Direct sums of finitely many copies of that group provide more examples. Together with subgroups of these groups, we have a continuum of examples. For non-abelian examples, note that the group of upper unitriangular $n$-by- $n$ rational matrices is nilpotent of class $n-1$ and of finite rank.

The following concept is a variation of one mentioned in [dlH 00, VII.40].

Definition 1. A group $G$ is uniformly locally of polynomial growth if there exist functions $A(d)$ and $t(d)$, such that each $d$-generator subgroup $H$ of $G$ satisfies $s_{H}(n) \leq A(d) n^{t(d)}$ (with respect to some set of generators).

The definition in [dlH 00] is slightly different. The term used is uniform polynomial growth, and $G$ is said to enjoy that property, if each $d$-subset $X$ of $G$ satisfies $\left|X^{n}\right| \leq$ $A(d) n^{t(d)}$. It follows from Theorem 3 below that the two concepts, de la Harpe's and ours, coincide. 
Definition 2. A group $G$ is uniformly locally nilpotent, or uniformly locally finite, if there exists a function $f(d)$, such that each $d$-generated subgroup of $G$ is nilpotent of class at most $f(d)$, or finite of order at most $f(d)$, respectively (in [Sh 93] the term strongly locally nilpotent is used).

Theorem 3. (1) $G$ is uniformly locally of polynomial growth if and only if there exist two functions $f(d)$ and $g(d)$, such that each $d$-generated subgroup of $G$ contains a nilpotent subgroup of class at most $f(d)$ and index at most $g(d)$.

(2) An extension of a uniformly locally nilpotent group by a uniformly locally finite group is uniformly locally of polynomial growth.

The proofs of these theorems depend, as in [Gr 81] and [VdDW 84a], on explicit, quantitative versions of Gromov's theorem.

Theorem 4. Let $G$ have polynomial growth; specifically, let $s_{G}(n) \leq C n^{d}$. Then $G$ contains a nilpotent subgroup of finite index and of class less than $\sqrt{2 d}$.

Surprisingly, the value $\sqrt{2 d}$, or indeed any explicit bound for the class of a finite index nilpotent subgroup, seems not to have appeared in the literature.

Theorem 5. Let numbers $C$ and $d$ be given. Then there exist numbers $k$ and $r$, such that if a finitely generated group $G$ satisfies $s_{G}(n) \leq C n^{d}$ for $n=1, \ldots, k$, then $G$ contains a nilpotent subgroup of class less than $\sqrt{2 d}$ and index at most $r$. The numbers $k$ and $r$ depend only on $C$ and $d$.

Except for the bound $\sqrt{2 d}$, this is stated on p. 71 of [Gr 81], and it is also Theorem 7.1 of [VdDW 84a]. In the latter reference it is shown that $k$ and $r$ can be computed effectively (though not efficiently) from $C$ and $d$.

Theorem 6. Let $G$ be a finitely generated group of polynomial growth of degree $d$. Then $G$ contains normal subgroups $N$ and $L$, such that $L \leq N, L$ is finite, $N / L$ is nilpotent, $\operatorname{cl}(N / L)<\sqrt{2 d}$, and $|G: N| \leq h(d)$.

In the case $d=1$ the proof will show that $G / L$ is either infinite cyclic or the infinite dihedral group. This is stated in [IS 87], where it is deduced from the theory of groups with two ends. It also follows from the results there that if $d=1$ in Theorem 5 , then one can take $k=r=2 C$.

The definition of uniform local polynomial growth in [dlH 00] modifies slightly the definition in [Bo 80] (there M. Bozejko forgot to introduce the coefficient $A(d)$ ). Bozejko defines also uniformly amenable groups, and shows that uniform local polynomial growth implies uniform amenability. For completeness we show that his argument applies in more generality. 
Definition 3. A group $G$ has uniformly locally subexponential growth if for each $d$ there exists a function $f_{d}(n)$, such that $\lim \inf f_{d}(n)^{\frac{1}{n}}=1$, and for each $d$-subset $X$ of $G$ we have $\left|X^{n}\right| \leq f_{d}(n)$.

Proposition 7. A group of uniformly locally subexponential growth is uniformly amenable.

While this seems stronger than Bozejko's result, we have to say that we do not have any examples of groups of uniform local subexponential growth but not of uniform local polynomial growth. In contrast to the case of ordinary subexponential growth, there are no residually finite groups of that type. Indeed a weaker assumption than subexponential growth suffices.

Proposition 8. Let $G$ be locally residually finite. Suppose that there exist some d and $n$, such that for each $d$-subset $X$ of $G$ we have $\left|X^{n}\right|<d^{n}$. Then $G$ is uniformly locally of polynomial growth. If $G$ itself is residually finite, it is an extension of a uniformly locally nilpotent group by a uniformly locally finite group.

This will be seen to follow from results of A. Shalev [Sh 93]. A similar argument occurs in [B1 98]. Note that if functions $f_{d}(n)$ as in Definition 3 exist, even satisfying the weaker condition $\liminf f_{d}(n)^{\frac{1}{n}}<d$, then the inequality $\left|X^{n}\right|<d^{n}$ holds for some $d$ and $n$ (and all $d$-subsets $X$ ). Thus we have

Corollary 9. A locally residually finite group of uniform local subexponential growth is uniformly locally of polynomial growth.

\section{Proofs}

Proof of Theorem 4. This is a converse of Bass' formula [Ba 72], giving the degree of polynomial growth of a nilpotent group. Let $G$ be a finitely generated nilpotent group, let $\left\{\gamma_{i}(G)\right\}$ be its lower central series, and let $r_{i}$ be the torsion-free rank of $\gamma_{i}(G) / \gamma_{i+1}(G)$, i.e. the number of infinite factors in a decomposition of $\gamma_{i}(G) / \gamma_{i+1}(G)$ as a direct product of cyclic groups. Bass' formula states that $G$ has polynomial growth of degree $d=\sum i r_{i}$. This does not yield a bound for the class of $G$ in terms of $d$, because some of the lower central factors may be finite. But it is well known that $G$ contains a torsion-free subgroup of finite index. Thus we assume that $G$ itself is torsion-free, and let $\delta_{i}(G) / \gamma_{i}(G)$ be the torsion subgroup of $G / \gamma_{i}(G)$ and $c=\operatorname{cl}(G)$. Then $\delta_{c+1}(G)=1$. It is well known that $\delta_{i}(G) / \gamma_{i}(G)$ is a finite group, and that $\left\{\delta_{i}(G)\right\}$ is a central series for $G$. By definition, the factors $\delta_{i}(G) / \delta_{i+1}(G)$ are torsion-free, and have torsion-free rank rank $r_{i}$. Because the class is exactly $c$, none of the factors $\delta_{i}(G) / \delta_{i+1}(G)$, for $i \leq c$, is trivial, i.e $r_{i} \geq 1$ for 
$i \leq c$. Thus $d=\sum_{1}^{c} i r_{i} \geq \frac{1}{2} c(c+1)$, yielding $c<\sqrt{2 d}$. Unless $G$ is cyclic, we have $r_{1} \geq 2$, therefore we actually have $d \geq \frac{1}{2} c(c+1)+1$.

The above proof establishes the following

Corollary 10. Let $G$ be a finitely generated torsion free nilpotent group, of class $c$ and growth degree $d$. Unless $G$ is cyclic, we have $d \geq \frac{c^{2}+c+2}{2}$.

Proof of Theorem 5. Suppose that for some pair $C, d$ the result does not hold. Then we can find groups $G_{i}, i=1,2, \ldots$, satisfying $s_{G_{i}}(n) \leq C n^{d}$, for $n=1, \ldots, i$, but $G_{i}$ does not contain a nilpotent subgroup of class less than $\sqrt{2 d}$ and index at most $i$. Substituting $n=1$ in the inequalities, we see that the growth functions are relative to sets of at most $C$ generators, and without loss of generality we assume that $C$ is an integer, and that each $G_{i}$ is generated by exactly $C$ elements, say $x_{i, 1}, \ldots, x_{i, C}$. Let $\mathcal{F}$ be a non-principal ultrafilter on $\mathbb{N}$, and form the corresponding ultraproduct $K$ of the groups $G_{i}$. Let $G$ be the subgroup of $K$ generated by the elements $x_{1}, \ldots, x_{C}$, whose representatives are the sequences $\left\{x_{i, 1}\right\}, \ldots,\left\{x_{i, C}\right\}$. Consider $s:=s_{G}(n)$, for some $n$, and the elements $y_{1}, \ldots, y_{s}$ of $G$ of length at most $n$. Write each $y_{j}$ as a word $w_{j}$ of minimal length in the generators. Then for any two distinct indices $j, l$ the set $T(j, l):=\left\{i \in \mathbb{N} \mid w_{j}\left(x_{i, k}\right) \neq w_{l}\left(x_{i, k}\right)\right\}$ lies in $\mathcal{F}$, and so does their intersection $T$ (the inequalities are in $G_{i}$ ). Therefore $T$ is infinite, and choosing $i \in T, i>n$, we see that $G_{i}$ has at least $s$ distinct elements of length at most $n$. By assumption, $s \leq C n^{d}$, which means that $G$ has polynomial growth, and contains a nilpotent subgroup $H$ of finite index $r$ and class $c<\sqrt{2 d}$.

Let $F$ be the free group on $C$ generators $a_{1}, \ldots, a_{C}$, and let $N$ be the inverse image of $H$ under the natural homomorphism from $F$ onto $G$. Then $|F: N|=r$, and in the natural homomorphism of $F$ onto $G_{i}, N$ maps to a subgroup $H_{i}$ of index at most $r$. Let $N$ be generated by elements $\left\{u_{v}\right\}, v=1, \ldots, t$, written as words $u_{v}$ in the generators. Then in $G$ the elements $u_{v}\left(x_{j}\right)$ generate $H$, and each commutator of weight $c+1$ in these generators is 1 . But that means that $\mathcal{F}$ contains the set of indices $i$ for which the same commutators, on the elements $u_{v}\left(x_{i, j}\right)$, are 1 (in $G_{i}$ ), and in particular this set of indices is infinite. But the elements $u_{v}\left(x_{i, j}\right)$ generate $H_{i}$, and therefore for infinitely many $i$ 's $G_{i}$ contains a nilpotent subgroup of class $c$ and index at most $r$, contradicting our choice of $G_{i}$.

Proof of Theorem 6. We know already that $G$ contains a normal nilpotent subgroup $K$ of finite index and of class less than $\sqrt{2 d}$. Consider the central series $\delta_{i}(K)$, defined as in the proof of Theorem 4, and for each $i \leq c:=\operatorname{cl}(K)$ write $C_{i}=$ $C_{G}\left(\delta_{i}(K) / \delta_{i+1}(K)\right)$, and let $N=\bigcap C_{i}$. Then $K \leq N . G / N$ is the group of automorphisms that $G$ induces on $\bigoplus \delta_{i}(G) / \delta_{i+1}(G)$, and therefore $|G: N| \leq h(d)$. By definition, we have $K / \delta_{c+1}(K) \leq Z_{c}\left(N / \delta_{c+1}(K)\right)$, and therefore $\gamma_{c+1}\left(N / \delta_{c+1}(K)\right)$ is finite, by [Ro 96, 14.5.1]. But $\delta_{c+1}(K)$, which is the torsion subgroup of $K$, is finite, hence $\gamma_{c+1}(N)$ is finite as well, and we are done. 
Proof of Theorem 1. Let $G$ satisfy condition (i). We remarked already that each finitely generated subgroup of $G$ is generated by $C$ elements. By Theorem 5, there exists a number $r$ such that each finitely generated subgroup $H$ contains a normal nilpotent subgroup $N$ of class less than $\sqrt{2 d}$ and index at most $r$. Let $A(H)$ be the set of such subgroups, and for each pair $H \leq K$ of finitely generated subgroups, consider the map $N \rightarrow N \cap H$ from $A(K)$ into $A(H)$. This defines an inverse system of finite sets and maps, and there exists a point in the inverse limit of that system. Let $\{N(H)\}$ be a point in the inverse limit, and write $N=\bigcup N(H)$. If $x, y \in N$, then $x \in N(H)$ and $y \in N(K)$ for some finitely generated subgroups $H$ and $K$. Write $L=\langle H, K\rangle$. Then $x, y \in N(L)$, therefore $x y \in N(L) \leq N$, and thus $N$ is a subgroup. Next, if $x \in N(H)$ and $y \in G$, then in the subgroup $L=\langle H, y\rangle$ we have $x^{y} \in N(L) \leq N$, and this shows that $N$ is normal. Let $c$ be the largest integer which is smaller than $\sqrt{2 d}$, and let $x_{1}, \ldots, x_{c+1} \in N$, say $x_{i} \in N\left(H_{i}\right)$. Then $x_{i} \in N(L)$, where this time $L$ is generated by all the $H_{i}$ 's, therefore $\left[x_{1}, \ldots, x_{c+1}\right]=1$, and $N$ is nilpotent of class $c$. Finally, if $z_{1}, \ldots, z_{r+1} \in G$, and $L=\left\langle z_{1}, \ldots, z_{r+1}\right\rangle$, then two of the $z_{i}$ 's lie in the same coset of $N(L)$, and therefore in the same coset of $N$. Thus $|G: N| \leq r$.

For the converse, suppose that $G$ is nilpotent-by-finite and of finite rank, and that each finitely generated subgroup of $G$ can be generated by $k$ elements. Let $H$ be a nilpotent subgroup, say of class $c$, of finite index $r$. Let $F_{k}$ be the free group of rank $k$, and let $N$ be the intersection of the kernels of all homomorphisms of $F_{k}$ into $G$. For each such homomorphism, with kernel $K$, say, let $L$ be the inverse image of $H$. Then $\left|F_{k}: L\right|=r$ and $\gamma_{c+1}(L) \leq K$. Let $M$ be the intersection of all subgroups of index $r$ in $F_{k}$. This is a subgroup of finite index, and $\gamma_{c+1}(M) \leq K$, for each $K$, therefore $\gamma_{c+1}(M) \leq N$. Thus $F_{k} / N$ is nilpotent-by-finite. Let $S$ be any finitely generated subgroup of $G$. Then $S$ can be generated by $k$ elements, hence it is a homomorphic image of $F_{k}$. In this homomorphism $N$ is in the kernel. Thus $S$ is an image of $G / N$, and the growth function of $G / N$ (which is polynomial) is an upper bound for the growth function of $S$. Thus all finitely generated subgroups of $G$ have a common polynomial upper bound for their growth functions.

Proof of Theorem 2. First assume that $G$ satisfies condition (1). By Theorem 6, each finitely generated subgroup $H$ contains a normal subgroup $N(H)$ of index at most $h(d)$ such that $\gamma_{c+1}(N(H))$ is finite, where $c=\lfloor\sqrt{2 d}\rfloor$. Let $A(H)$ be the set of such subgroups, and for each pair $H \leq K$ of finitely generated subgroups, consider the map $N \rightarrow N \cap H$ from $A(K)$ into $A(H)$. This defines an inverse system of finite sets and maps, and there exists a point in the inverse limit of that system. If $\{N(H)\}$ is that point, then, as in the proof of Theorem $1, N:=\bigcup N(H)$ is a normal subgroup of index at most $h(d)$. A finitely generated subgroup of $\gamma_{c+1}(N)$ is contained in $\gamma_{c+1}(N(H))$, for some finitely generated subgroup $H$, and therefore $\gamma_{c+1}(N)$ is locally finite. $\delta\left(N / \gamma_{c+1}(N)\right)$ is also locally finite, and we take $L$ to be the subgroup of $G$ satisfying $L / \gamma_{c+1}(L)=\delta\left(N / \gamma_{c+1}(N)\right)$. Each finitely generated 
subgroup $H$ of $N$ has Hirsch rank at most $d$, by Bass' formula, and looking in the series $\delta_{i}(H / H \cap L)$, with free abelian factors, shows that $H / H \cap L$ can be generated by $d$ elements. Finally, $G$ is locally Noetherian, because each finitely generated subgroup is nilpotent-by-finite.

Conversely, assume that $G$ satisfies (2). Let $H$ be a finitely generated subgroup of $G$. Since $H$ is Noetherian, $H \cap L$ is finitely generated, and since $L$ is locally finite, $H \cap L$ is finite. Then the series $H \cap L \triangleleft H \cap N \triangleleft H$, with the first and last terms finite and the middle one nilpotent, shows that $H$ has polynomial growth. Moreover, the middle link has a central series of length $c$ with factors that can be generated by $k$ elements, for some $c$ and $k$, therefore Bass' formula shows that the degree of $H$ is at most $c k$.

We now come to Gromov's result mentioned in the introduction. The following is an obvious corollary of Theorem 2 .

Corollary 11. A torsion-free group has all its finitely generated subgroups of polynomial growth of bounded degree if, and only if, it is virtually nilpotent of finite rank.

This implies a converse to a remark made following the statement of Theorem 2 above.

Corollary 12. A group as in Corollary 11 is a subgroup of $\operatorname{GL}(n, \mathbb{Q})$, for some $n$.

Indeed, it suffices to show this for nilpotent groups of finite rank, and for such groups this is the Corollary on p. 25 of [We 73].

Proof of Theorem 3. (1) One direction follows immediately from Theorem 5. Conversely, if the functions $f(d)$ and $g(d)$ exist, consider in the free group of rank $d$ the intersection $H$ of all subgroups of index at most $g(d)$, then all $d$-generated subgroups of $G$ are images of $H / \gamma_{f(d)}(H)$, and their growth functions are bounded by the growth function of the latter group. Item (2) is proved similarly.

Proof of Proposition 7. First we recall that $G$ is uniformly amenable if for each $\varepsilon>0$ there exists a function $q_{\varepsilon}(n)$ such that for each finite subset $E$ of $G$ there exists a finite subset $K$ such that $|E K| \leq(1+\varepsilon)|K|$ and $|K| \leq q_{\varepsilon}(|E|)$. Now we repeat Bozejko's argument. Suppose that $G$ is of uniform local sub-exponential growth, but it is not uniformly amenable. Then there exist some $\varepsilon$ and some number $k$, such that for each $r$ we can find a $k$-subset $E$ of $G$, such that for all $s$-subsets $K$ of $G$, with $s \leq r$, we have $|E K|>(1+\varepsilon)|K|$. Choose $r=k^{n}$. Then for the corresponding $E$ we take as $K$ the subsets $E^{m}$, with $m=n, n-1, \ldots$, and obtain $\left|E^{n+1}\right|>(1+\varepsilon)^{n} k$. Then $f_{k}(n+1)>(1+\varepsilon)^{n} k$, and $\liminf f_{k}(n)^{\frac{1}{n}} \geq 1+\varepsilon$, a contradiction. 
Proof of Proposition 8. Groups for which $d$ and $n$ as in the proposition exist, are termed in [Sh 93] collapsing. According to the main result there, residually finite collapsing groups are (uniformly locally nilpotent)-by-(finite exponent). Moreover, if $n$ and $d$ are given, then there exist functions $f(e, k)$ and $g(k)$, such that $G$ contains a normal subgroup $N$ such that $\exp (G / N) \mid g(r)$, and each $e$-generated subgroup of $N$ is nilpotent of class at most $f(e, r)$, where $r=\max (d, n)$. This, combined with E. Zelmanov's solution of the Restricted Burnside Problem, proves the proposition.

\section{Concluding remarks}

The inequality $d \geq \frac{1}{2}\left(c^{2}+c+2\right)$, which was obtained in Corollary 10 , is best possible. To see this, start with a free abelian group of rank $c$, with basis $x_{1}, \ldots, x_{c}$, and extend it by an element $x$ of infinite order such that $x_{c}^{x}=x_{c}$ and $x_{i}^{x}=x_{i+1}$ for $i<c$. The extended group is torsion-free, nilpotent of class $c$, and degree of growth $d=\left(c^{2}+c+2\right) / 2$.

Next, given any finite subgroup $F$ of $\operatorname{GL}(d, \mathbb{Z})$, the split extension $G$ of $\mathbb{Z}^{d}$ by $F$ has polynomial growth of degree $d$. Let $N \triangleleft G$ be nilpotent, and let $T$ be the torsion subgroup of $N$. Then $T \triangleleft G$, and $\mathbb{Z}^{d} \cap T=1$, therefore $T$ centralizes $\mathbb{Z}^{d}$, which implies $T=1$, and in particular $N \cap F=1$ and $|G: N| \geq|F|$. Thus $h(d)$ is the best possible bound for the index of $N$ in Theorem 6 . But it is not the best possible bound for the index of a nilpotent subgroup in the situation of Corollary 11. Indeed, if $F$ above is a maximal finite subgroup of $\operatorname{GL}(d, \mathbb{Z})$, it contains the matrix $-I$. If now $G$ is an extension of $\mathbb{Z}^{d}$ by $F$, and $x \in G$ maps onto $-I$ (modulo $N$ ), then $x^{2} \in N$, and then $x^{2}$ is both inverted and fixed under conjugation by $x$, and thus $x^{2}=1$ and $G$ is not torsion-free. It may be of interest to find the best possible value for the index in a torsion-free group. As for $h(d)$, its finiteness was proved by H. Minkowski, who determined the 1.c.m. of the orders of all finite subgroups of $\operatorname{GL}(d, \mathbb{Z})$ ([Mi 87]; for a modern treatment see [Fe 97] or [GL 06]). The value of $h(d)$ was given by W. Feit, relying on results of B. Weisfeiler ([Fe 95], [We 84], [We 94]; see also [Fr 97]). Except for several very small values of $d$, we have $h(d)=2^{d} \cdot d$ !. That value is achieved by the group of monomial matrices with entries \pm 1 (the determination of $h(d)$ applies the classification of the finite simple groups).

One possible way to find groups of locally polynomial growth may be the following. By a famous theorem of G. Higman, B.H .Neumann, and H. Neumann, each countable group is a subgroup of a two-generator group. Let the countable group $G$ be a subgroup of the finitely generated group $H$. We can count the elements of $G$ according to their length in $H$. It is then possible that the growth function of $G$ defined in this manner is polynomial, and then Theorem 2 applies. I have not seen any investigations along these lines. Another possible situation is this: suppose that $G$ is 
a subgroup of an infinitely generated group $H$, and that a set of generators for $H$ is given. Then we can still define the length of each element relative to these generators. In $H$ there may be infinitely many elements of each length, but it may happen that $G$ contains only finitely many elements of each length. Note that in that case the exponential bound for the number of elements of each length does not necessarily applies. Again I know of no investigations of such situations, not even some examples.

\section{References}

[Ba 72] H. Bass, The degree of polynomial growth of finitely generated nilpotent groups. Proc. London Math. Soc. (3) 25 (1972), 603-614. Zbl 0259.20045 MR 0379672

[B1 98] S. Black, Asymptotic growth of finite groups. J. Algebra 209 (1998), 402-426. Zbl 0922.20036 MR 1659911

[Bo 80] M. Bożejko, Uniformly amenable discrete groups. Math. Ann. 251 (1980), 1-6. Zbl 0422.43001 MR 583820

[dlH 00] P. de la Harpe, Topics in geometric group theory. Chicago Lectures in Math., University of Chicago Press, Chicago 2000. Zbl 0965.20025 MR 1786869

[Fe 95] W. Feit, The orders of finite linear groups. Preprint 1995.

[Fe 97] W. Feit, Finite linear groups and theorems of Minkowski and Schur. Proc. Amer. Math. Soc. 125 (1997), 1259-1262. Zbl 0879.20003 MR 1376761

[Fr 97] S. Friedland, The maximal orders of finite subgroups in $G L_{n}(\mathbf{Q})$. Proc. Amer. Math. Soc. 125 (1997), 3519-3526. Zbl 0895.20039 MR 1443385

[Gr 81] M. Gromov, Groups of polynomial growth and expanding maps. Inst. Hautes Études Sci. Publ. Math. (1981), 53-73. Zbl 0474.20018 MR 623534

[GL 06] R. M. Guralnick and M. Lorenz, Orders of finite groups of matrices. In Groups, rings and algebras, Contemp. Math. 420, Amer. Math. Soc., Providence, RI, 2006, 141-161. Zbl 05150289 MR 2279238

[IS 87] W. Imrich and N. Seifter, A bound for groups of linear growth. Arch. Math. (Basel) 48 (1987), 100-104. Zbl 0584.20025 MR 878419

[Ju 71] J. Justin, Groupes et semi-groupes à croissance linéaire. C. R. Acad. Sci. Paris Sér. A-B 273 (1971), A212-A214. Zbl 0218.20050 MR 0289689

[Mi 87] H. Minkowski, Zur Theorie der positiven quadratische Formen. J. Reine Angew. Math. 101 (1887), 196-202; reprinted in Gesammelte Abhandlungen, vol. I, B.G. Teubner, Leipzig 1911, 212-218.

[Ro 96] D. J. S. Robinson, A course in the theory of groups, 2nd ed. Grad. Texts in Math. 80, Springer-Verlag, New York 1996. Zbl 0836.20001 MR 1357169

[Sh 93] A. Shalev, Combinatorial conditions in residually finite groups, II. J. Algebra 157 (1993), 51-62. Zbl 0814.20023 MR 1219658 
[VdDW 84a] L. van den Dries and A. J. Wilkie, Gromov's theorem on groups of polynomial growth and elementary logic. J. Algebra 89 (1984), 349-374. Zbl 0552.20017 MR 751150

[VdDW 84b] L. van den Dries and A. J. Wilkie, An effective bound for groups of linear growth. Arch. Math. (Basel) 42 (1984), 391-396. Zbl 0567.20016 MR 0756689

[We 73] B. A. F. Wehrfritz, Infinite linear groups. Ergeb. Math. Grenzgeb. 76, SpringerVerlag, New York 1973. Zbl 0261.20038 MR 0335656

[We 84] B. Weisfeiler, Post-classification version of Jordan's theorem on finite linear groups. Proc. Nat. Acad. Sci. U.S.A. 81 (1984), 5278-5279. Zbl 0542.20026 MR 758425

[We 94] B. Weisfeiler, On the size and structure of finite linear groups. Preprint 1994.

[Wo 68] J. A. Wolf, Growth of finitely generated solvable groups and curvature of Riemanniann manifolds. J. Differential Geometry 2 (1968), 421-446. Zbl 0207.51803 MR 0248688

Received January 29, 2007; revised June 28, 2007

A. Mann, Einstein Institute of Mathematics Givat Ram, The Hebrew University of Jerusalem, Jerusalem, 91904, Israel

E-mail: mann@math.huji.ac.il 